\title{
Comparaison Quantitative Et Qualitative Des Huiles Essentielles De Rosmarinus Officinalis Obtenues Par Différentes Méthodes
}

\begin{abstract}
Taha EL Kamli
Laboratoire des Essais Biologiques. Université Ibn Tofaïl Kénitra (Maroc).

Faouzi Errachidi

Laboratoire de Physiopathologie. Génétique Moléculaire et biotechnologie.

Faculté des Sciences Aïn Chock Casablanca (Maroc).

\section{Noureddine Eloutassi}

Laboratoire science de la vie. Centre Régional des Métiers de l'Education et de la Formation. Fès (Maroc).

\section{Houmane Majid}

Société HERBADIS : production, distribution et vente des huiles essentielles, et des arômes alimentaires. Fès (Maroc).

\section{Rachida Chabir}

Laboratoire de Physiopathologie, Nutrition et Environnement. Faculté de médecine et de pharmacie Fès (Maroc).

\section{Abdellatif Bour}

Laboratoire des Essais Biologiques. Équipe de la Transition Alimentaire et Nutritionnelle (ETAN), Université Ibn Tofaïl Kénitra (Maroc).
\end{abstract}

\section{Abstract}

Rosmarinus officinalis is among the most abundant medicinal and aromatic plants in Morocco. There are several factors that influence the yield of quality and quantity of essential oils of this plant. This work focuses on the study, characterization and comparison of the chemical composition of essential oils of Rosmarinus officinalis. The raw material studied is originally from the central part of northern Morocco (Skoura M'Daz region, Eastern Middle Atlas). The essential oils of rosemary were obtained by three different extraction methods : DA: Artisanal distillation, the essential oils were obtained directly from small cooperatives using a very traditional (nonindustrial) method and based on the technique of Steam distillation; DI: Industrial distillation which is also based on Steam distillation; DC: hydrodistillation by clevenger apparatus Laboratory. The physicochemical characterization of essential oils focuses on the search for the yield of $\%$ 
essential oil with regard to the dry rosemary biomass, density, refractive index and rotatory power. The chemical analyzes were carried out by gas chromatography (GC), they identified 11 components representing more than $99.64 \%$ of the essential oil and indicate that the chemotype is 1.8 cineole and is varied according to the method (DI: $48.83 \%$, DA: $41.28 \%$ and DC: $51.77 \%$ ). In conclusion we have shown a quantitative and qualitative loss of essential oils due to the extraction method of essential oils, which has direct repercussions on the economy of rural Morocco and consequently on their sustainable development.

Keywords: Rosmarinus officinalis, Essential oil, Extraction, Middle Atlas, Morocco

\section{Résumé}

Rosmarinus officinalis est parmi les plantes médicinales et aromatiques les plus abondantes au Maroc. Il existe plusieurs facteurs qui influencent le rendement en qualité et en quantité des huiles essentielles de cette plante. Le présent travail s'intéresse à l'étude, à la caractérisation et à la comparaison de la composition chimique des huiles essentielles de Rosmarinus officinalis. La matière première étudiée est d'origine de la partie centrale du nord de Maroc (région Skoura M' Daz, Moyen Atlas oriental). Les huiles essentielles du romarin ont été obtenues par trois méthodes d'extraction différentes : DA : Distillation artisanale, les huiles essentielles ont été obtenues directement des petites coopératives utilisant une méthode très artisanale (non industrielle) et basant sur la technique de vapodistillation; DI : Distillation industrielle qui se base aussi sur la vapodistillation DC: Hydrodistillation par Clevenger de laboratoire. La caractérisation physicochimique des huiles essentielles se concentre sur la recherche du rendement en \% d'huile essentielle par apport à la biomasse sèche du romarin, sur la densité, sur l'indice de réfraction et sur le pouvoir rotatoire. Les analyses chimiques ont été effectuées par chromatographie en phase gazeuse (CPG), elles ont permis d'identifier 11 composants représentant plus de $99.64 \%$ de l'huile essentielle et indiquent que le chémotype est le 1,8 cinéole et se varié selon la méthode d'obtention (DI : $48.83 \%$, DA : $41.28 \%$ et DC : $51.77 \%$ ). En conclusion, nous avons montré une perte quantitative et qualitative des huiles essentielles due à la méthode d'extraction des huiles essentielles ce qui se répercute directement sur l'économie des régions rurale marocaine et par conséquent sur leurs développement durable.

Mots-Clefs: Rosmarinus officinalis, Huile essentielle, Extraction, Moyen Atlas, Maroc 


\section{Introduction}

Le romarin (Rosmarinus officinalis L.) est une plante aromatique et médicinale, arbrisseau touffu, xérophyte, toujours vert de 1 à $2 \mathrm{~m}$ de hauteur selon les régions de répartition. Morphologiquement, il se modifie selon plusieurs facteurs du milieu. Il fait partie des espèces végétales des écosystèmes terrestres qui se présentent à l'état sauvage dans les zones littorales près de la mer, dans les milieux continentaux au climat semi humide, sec et arides (Boudy, 1948), (Granger, 1976), (Herrera, 2005),

(Mehdioui, 2007), (Eloutassi, 2013), (Mateu, 2013).

$\mathrm{Au}$ Maroc, le romarin se développe en association avec le pin d'Alep (Pinus halepensis) et le pin maritime (Pinus mogrebiana), Il occupe des surfaces importantes dans le Rif occidental (Benadib, 1982)

En 2014 le Maroc a exporté près de 8000 tonnes de matière sèche du romarin vers le monde (L'Etablissement Autonome de Contrôle et de Coordination des Exportations 2014). Elles sont utilisées pour extraire les principes actifs recherchés en industrie pharmaceutiques, cosmétique et alimentaire.

Le romarin renferme une huile essentielle à laquelle il doit ses propriétés intéressantes; il est connu pour ses propriétés antioxydantes (Wang, 2008), antimicrobiennes (Burt, 2004), analgésiques (A.L. Martínez, 2012), anti-inflammatoires (M. Minaiyan, 2011) et anti-ulcérogènes (P.C. Dias, 2000). Il stimule le fonctionnement de la vésicule biliaire, il agit sur les fermentations intestinales et sur les douleurs abdominales qu'elles entraînent, il calme aussi la toux et contribue au confort de l'asthmatique. Il est tonicardiaque, hypotenseur décongestionnant veineux, régulateur hépatique etc. La production nationale en matière des huiles essentielles du romarin est estimée à environ 40 tonnes (L'Etablissement Autonome de Contrôle et de Coordination des Exportations 2014).

La valorisation du romarin au Maroc fait surgir plusieurs problématiques qui nécessitent une bonne réflexion sur les conditions d'extraction des huiles essentielles. L'estimation qualitative et quantitative de cette plante de haute valeur ajoutée peut offrir des potentialités intéressantes d'exploitation rationnelles au Maroc.

L'objectif de ce travail est l'évaluation qualitative et quantitative des composés majoritaires des huiles essentielles du romarin spontané extraites par des méthodes d'extractions artisanales et industrielles. Pour cette étude, nous avons utilisés le site Skoura M'Daz pour l'échantillonnage (origine) du romarin. Le but de cette analyse est d'évaluer la qualité des huiles essentielles de romarin extraites par les deux méthode, afin de localiser les imperfections et d'améliorer qualitativement et quantitativement le rendement en huile essentielle et par conséquent, contribuer à la mise à 
niveau du secteur pour devenir plus compétitif et participer massivement au développement durable des régions rurales marocaines.

\section{Matériels et méthodes}

\section{Zone d'Etude}

Le site Skoura M'Daz est situé dans la partie central du nord de Maroc, entre $33^{\circ} 10^{\prime}$ et $33^{\circ} 30^{\prime}$ de l'attitude Nord et de $4^{\circ} 10^{\prime}$ et $4^{\circ} 50^{\prime}$ de longitude Est. La figure 1 montre la position géographique du site d'étude et la figure 2 représente des vues générales panoramiques de ce site.

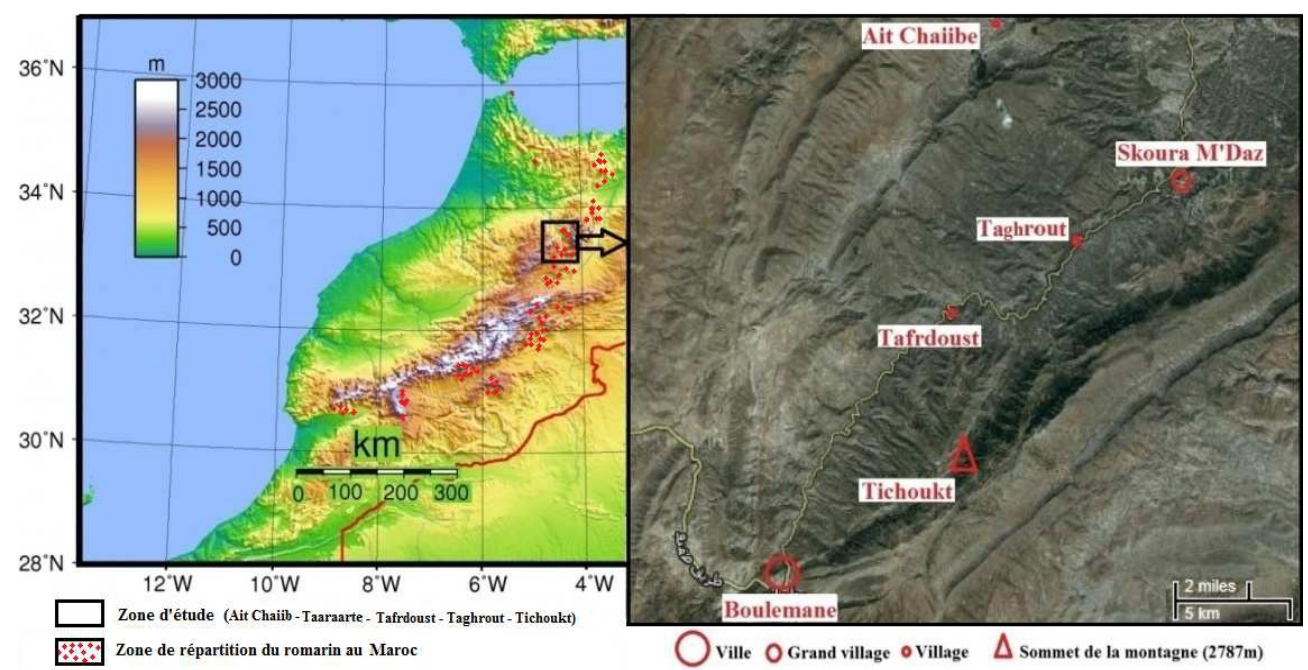

Figure 1 : Répartition de Rosmarinus officinalis au Maroc et situation géographique du site d'étude Skoura M'Daz

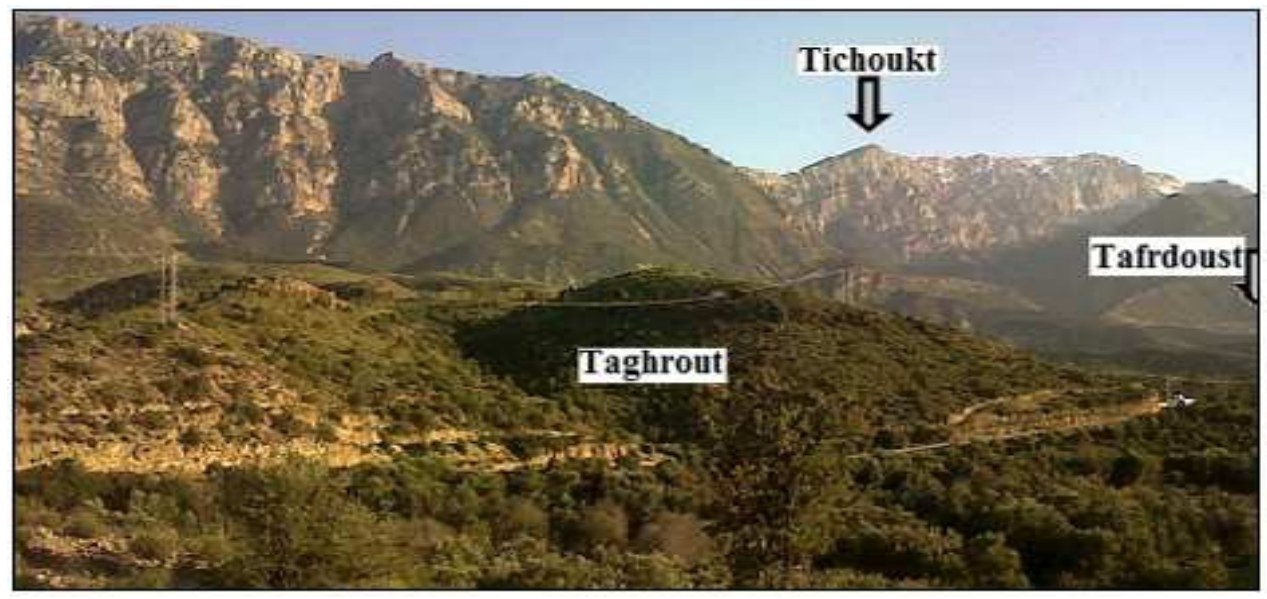

Figure 2: Vue générale panoramique de site d'étude Skoura M'Daz (les sites du romarin sont Taghrout, et Taferdoustet) 


\section{Condition opératoires et échantillonnage}

Les échantillons du romarin ont été collectés pendant le mois de Mai 2014 dans le site Skoura M'Daz. Nous avons choisi la même placette de récolte pour toutes les études. La placette étudiée du site est la région Taghrout/Taferdoustet (Figure 2) où le romarin se reparti d'une façon très condensé.

Les feuilles, les fleurs et les sommités fleuries du romarin ont été fraîchement récoltées. Elles sont séchées à l'ombre, étalées sur des étagères à température ambiante $\left(27^{\circ} \mathrm{C}\right)$ et retournés afin d'éviter la formation éventuelle de moisissures induites par l'humidité. Les parties aériennes sont ensuite triées avec soin et soumises à l'extraction par les trois techniques de distillation (DA, DC, DC). L'optimisation des conditions opératoires de l'extraction par hydrodistillation ont été étudiées postérieurement (Eloutasi 2004). Le taux d'humidité du matériel végétal a été déterminé avant la distillation, pour cela la matière végétale, recueillie, a été séchée à l'air libre, à l'ombre jusqu'à la stabilisation de son poids (7 jours). Les rendements en HE sont exprimés par rapport à la matière sèche $(\mathrm{g} / 100 \mathrm{~g})$. La pression de distillation utilisée dans les trois techniques est effectuée sous des conditions atmosphériques normales.

\section{Extraction}

Les tests qualitatifs et quantitatifs du romarin ont été effectués sur des huiles essentielles extraites par trois techniques différentes de distillation :

- $\quad$ Par entrainement à la vapeur d'eau dans une unité industrielle (DI) de $2 \mathrm{~m} 3$ (Figure 3).

- Par entrainement à la vapeur d'eau dans un appareillage artisanale (DA) utilisé par plusieurs coopératives marocaine (Figure 4).

- Par hydrodistllation de Clevenger (Figure 5), un appareillage de laboratoire (DC).

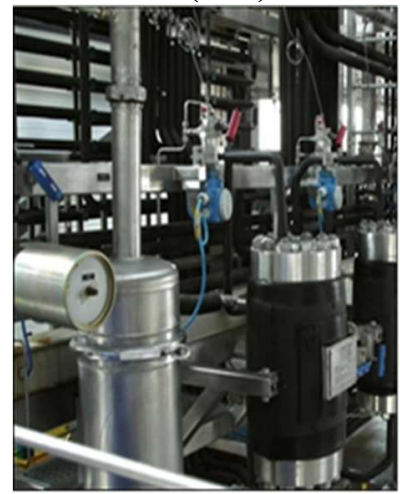

A

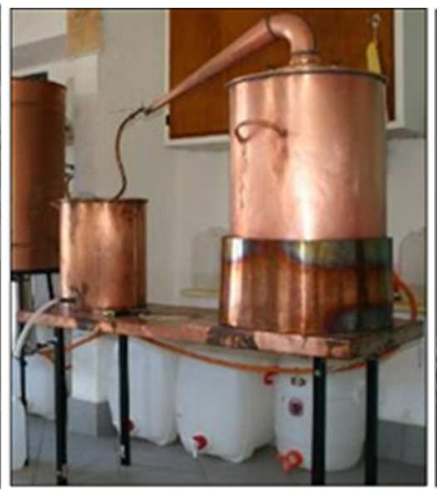

B

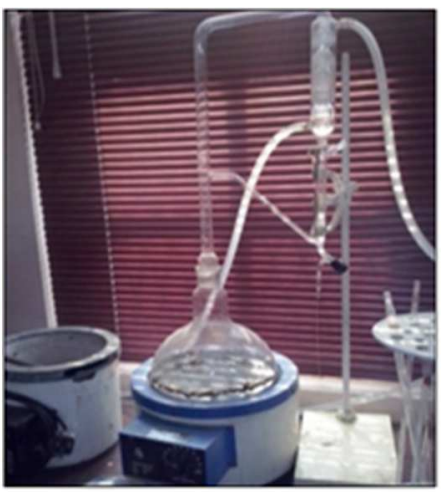

$\mathrm{C}$

Figure 3 les trois types de distillations étudiées dans cette recherche (A : Distillation industrielle (DI), B : Distillation artisanale (DA) et C : Hydrodistillation par Clevenger (DC). 


\section{Caractéristiques physico-chimiques}

Nous avons déterminé les caractéristiques physico-chimiques et le rendement des huiles essentielles obtenues par les trois différentes méthodes de distillation DI, DA, DC selon le protocole décrit dans la pharmacopée européenne 2014. Ces caractéristiques physico-chimiques recherchées sont : la Densité (densimètre type METTLER TOLEDO 30 PX), le Pouvoir Rotatoire (Polarimètre ATAGO AP300) et l'Indice de Réfraction (réfractomètre type NAR-1TLIQUID).

\section{Analyse par chromatographie gazeuse}

Les analyses chromatographiques ont été effectuées par chromatographie en phase gazeuse « $\mathrm{CPG}$ » à régulation électronique de pression de type Perkin Elmer (Clarus 580). La colonne utilisée est capillaire de silice fondue, longueur $30 \mathrm{~m}$, diamètre de $0,25 \mathrm{~mm}$. Le gaz vecteur est l'hélium avec un débit de 1,0 $\mathrm{ml} / \mathrm{min}$. La température de la colonne est programmée automatiquement à $50^{\circ} \mathrm{C}$ pendant $10 \mathrm{~min}$, puis de 50 à $200^{\circ} \mathrm{C}$ durant $75 \mathrm{~min}$ et enfin à $200^{\circ} \mathrm{C}$ pour $25 \mathrm{~min}$. Les températures de l'injecteur et du détecteur gardent une valeur constante de $200^{\circ} \mathrm{C}$. L'identification des composants a été faite par co-injection de composés authentiques de référence.

\section{Résultats et discussion}

\section{Caractéristiques physico-chimiques}

L'huile essentielle obtenue par hydrodistillation des parties aériennes de Rosmarinus officinalis avait une couleur jaune clair et une odeur forte. Comme le montre le tableau 1 , les caractères physico-chimiques des trois huiles essentielles DI, DA, et DC du romarin sont différentes. Le rendement obtenu par DC est le plus élevé 2.4\%, suivi par la DI $2.2 \%$ et DA $2.1 \%$, la densité varie entre 0.900 et $0.905, l^{\prime}$ 'indice de réfraction entre 1.466 et 1.469 et l'angle de rotation entre $-0.3^{\circ}$ et $+0.4^{\circ}$. Mais malgré cette différence les échantillons des huiles essentielles DI, DA et DC sont conformes aux normes décrites par la pharmacopée Européenne 2014.

La détermination des propriétés physico-chimiques est une étape importante pour évaluer la qualité des huiles essentielle du romarin mais dans notre cas reste insuffisante pour relever la différence entre les trois méthodes d'extraction DI, DA, et DC. Il est donc nécessaire de la compléter par des analyses chromatographiques GC.

Tableau 1 : Paramètres physico-chimiques des huiles essentielles testées*

\begin{tabular}{ccccc}
\hline Echantillon & $\begin{array}{c}\text { Rendement en } \\
\mathrm{ml} / 100 \mathrm{~g}\end{array}$ & Densité & $\begin{array}{c}\text { Indice de } \\
\text { réfraction }\end{array}$ & $\begin{array}{c}\text { Ongle de rotatoire } \\
\left({ }^{\circ}\right)\end{array}$ \\
\hline DI & $2.25 \pm 0.08$ & $0.898 \pm$ & $1.468 \pm 0.037$ & +0.3 \\
& & 0.026 & & \\
DA & $2.14 \pm 0.13$ & $0.898 \pm$ & $1.469 \pm 0.042$ & +1.2
\end{tabular}


0.063

DC

$2.41 \pm 0.10$

$0.901 \pm$

$1.466 \pm 0.066$

$+2.4$

Norme**

Min 1.20

0.895 à

1.464 à 1.473

$-5^{\circ} \grave{a}+8^{\circ}$

0.920

*Valeur moyenne de 3 essais \pm Ecart type. **Pharmacopée européenne Ph. Eur., 2014.

Edition 8.3 DI : distillation industrielle, DA distillation artisanale et DC : Hydrodistillation par Clevenger de laboratoire.

\section{Composition chimique}

La composition chimique des huiles essentielle de Rosmarinus Officinalis obtenus par les trois méthodes DI, DA, et DC est décrite dans le tableau 2. Les résultats d'analyse par chromatographie en phase gazeuse des huiles essentielle ont été démontrés dans les figures 6, 7 et 8.

Tableau 2 : Composés majoritaires des huiles essentielles étudiées extraites par les trois types de distillation (DI, DA et DC)

\begin{tabular}{cccc}
\hline Composés $* *$ & DI $(\%)$ & DA $(\%)$ & DC $(\%)$ \\
\hline Alpha-pinène & $10,66 \pm 0.98$ & $11,27 \pm 0.76$ & $9,84 \pm 0.57$ \\
Camphène & $4,71 \pm 0.34$ & $4,53 \pm 0.28$ & $4,27 \pm 0.39$ \\
Beta-pinène & $3,25 \pm 0.01$ & $8,11 \pm 0.17$ & $0,10 \pm 0.00$ \\
Beta-myrcène & $2,40 \pm 0.04$ & $1,25 \pm 0.01$ & $1,94 \pm 0.03$ \\
Cineole & $48,83 \pm 2.13$ & $41,28 \pm 2.52$ & $51,77 \pm 1.97$ \\
p-cymène & $2,44 \pm 0.19$ & $2,23 \pm 0.25$ & $0,51 \pm 0.09$ \\
Camphre & $17,35 \pm 1.48$ & $22,82 \pm 1.96$ & $22,31 \pm 1.03$ \\
Acetate de Bornyle & $4,68 \pm 0.06$ & $5,53 \pm 0.11$ & $1,02 \pm 0.05$ \\
Alpha-terpinéole & $3,51 \pm 0.05$ & $1,72 \pm 0.04$ & $5,04 \pm 0.07$ \\
Bornéole & $1,64 \pm 0.03$ & $0,96 \pm 0.02$ & $2,80 \pm 0.01$ \\
Verbenone & $0,17 \pm 0.01$ & $0,06 \pm 0.01$ & $0,11 \pm 0.01$ \\
Total & 99,64 & 99,75 & 99,70
\end{tabular}

*Valeur moyenne de 3 essais \pm Ecart type **Les composés sont cités selon leur ordre d'élution

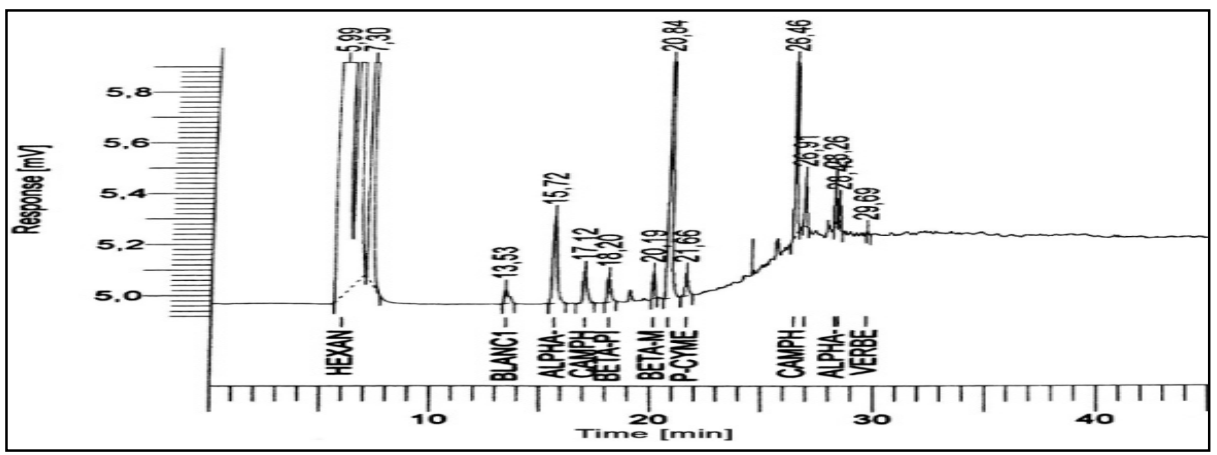

A 


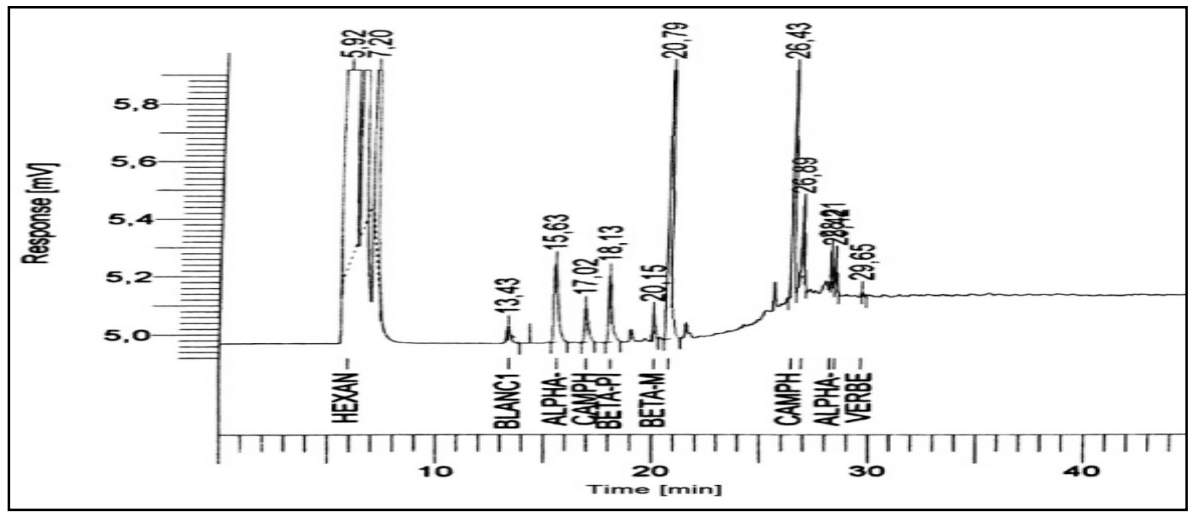

B

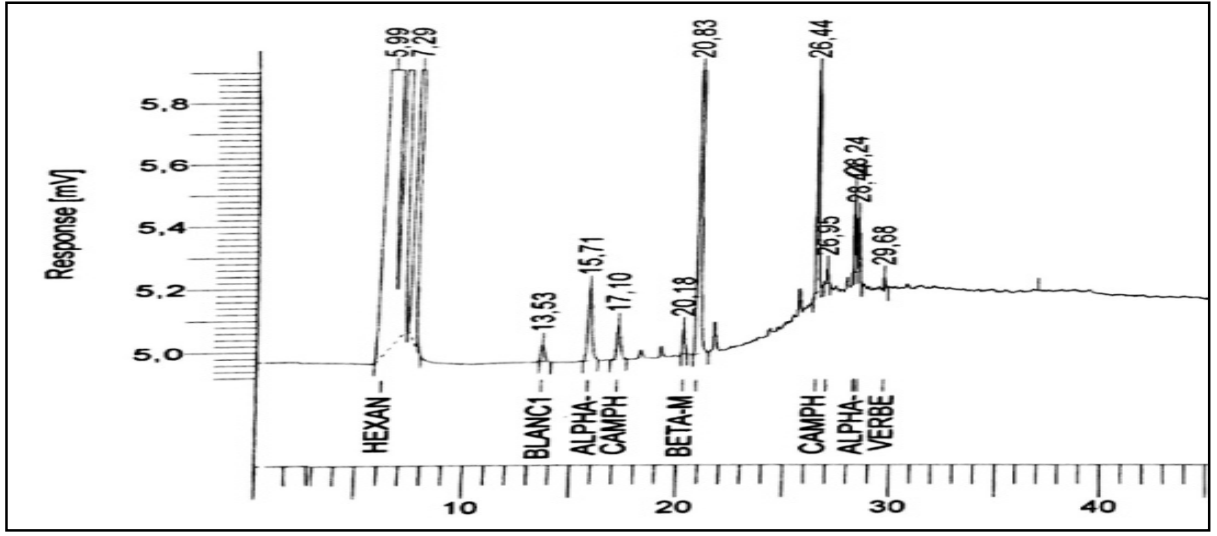

C

Figure 4 : Chromatogramme par chromatographie en phase gazeuse (CPG) des huiles essentielles extraites par (A : Distillation industrielle, B : Distillation artisanale (DA) et C : Hydrodistillation par Clevenger (DC).

Le composé majoritaire dans les 3 huiles essentielles étudiés est le 18 cinéole (DI : $48.83 \%$, DA : $41.28 \%$ et DC : $51.77 \%$ ) suivi par le camphre (DI : $17,35 \% ;$ DA : $22,82 \%$; DC : 22,31\%) et l'Alpha-pinène (DI : $10,66 \%$; DA : $11.27 \%$; DC : $9.84 \%$ ). Nous constatons que la nature des constituants est la même pour les trois types d'huile essentielles, et que les mono terpènes représentent la totalité des composés détectés avec la domination des monoterpènes Oxygénés $(72 \%$ à $83 \%)$ contre les hydrocarbures monoterpéniques (16\% à 27\%); cependant, la teneur des composés diffèrent selon la méthode d'extraction.

L'huile essentielle du romarin extraite par la méthode de distillation industrielle est la plus riche en Camphène (4.71\%), p-Cymène $(2.44 \%)$ et en Beta-myrcène $(2.40 \%)$. Par contre celle obtenue par la méthode artisanale présente une teneur supérieur en Camphre (22.82\%), Alpha pinène (11.27), Béta-pinène $(8.11 \%)$ et en Acetate de Bornyle (5.53\%). Cependant, la distillation du romarin au niveau du laboratoire par appareil Clevenger a 
donné une huile essentielle plus riche en 1,8-Ciéole (51.77\%), Alphaterpinéole (5.04\%), et Bornéole (2.80\%).

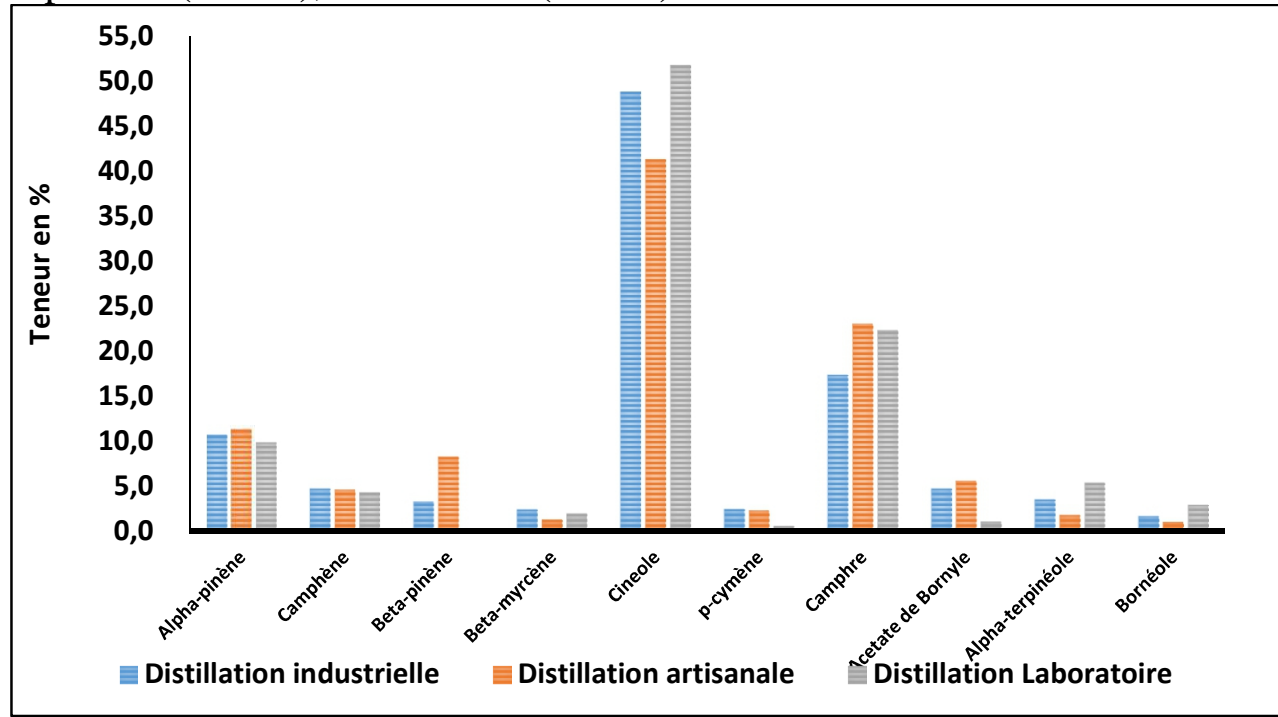

Figure 9 : Comparaison de la composition chimique des huiles essentielles du Rosmarinus officinalis DI, DA et DC

Comparativement, l'huile essentielle des romarins marocains obtenus par hydrodistillation présentent une teneur importante en l'un des 3 composés : $\alpha$-pinène $(37,0-40,0 \%$, Rabat), cinéole $(58,7-63,7 \%$, El Ateuf), camphre (41,7-53,8\%, Taforhalt) (Elamrani, 2000).

\section{Conclusion}

Les analyses des huiles essentielles par chromatographie en phase gazeuse montrent que les caractéristiques physico-chimiques, les compositions chimiques et les composés majoritaires sont différents et variables selon les méthode de l'extraction utilisée. Cette variation peut être très intéressante lors de la recherché industrielle d'un composé en qualité et en quantité et la méthode de sa distillation.

En perspective, le secteur des biotechnologies de productions végétales se trouve en pleine mutation et à l'affût des innovations technologiques. Le Maroc est caractérisé par une richesse très importante en plantes médicinales et aromatiques. Nous devons donc, relever des défis majeurs pour des usages variés principalement alimentaire, médicinale et aromatique. Dans un cadre d'objectifs et de contraintes socio-économiques et dans la perspective d'une agriculture contribuant au développement durable des territoires ruraux, Il est nécessaire de former des praticiens hautement spécialisés dans les Sciences du Végétal et leurs applications agronomiques, 
et ayant la maîtrise des procédés biotechnologiques de la production végétale.

\section{References :}

1. A.L. Martínez, M.E. González-Trujano, M. Chávez, F. Pellicer (2012). Antinociceptive effectiveness of triterpenes from rosemary in visceral nociception $J$ Ethnopharmacol, 142, pp. 28-34

2. BENADIB, A. (1982). Etude Phytoécologique, biogéographique et dynamique des associations et séries sylvatiques du Rif occidental (Maroc). Thèse Univ. Droitj Econ., Sc., Aix Marseille III.

3. Boudy P. (1948). Economie forestière nord-africain, tome I : milieu physique et milieu humain. Paris $\mathrm{V}^{\mathrm{e}}$, Edition Larose, p 125-216.

4. Burt, S., (2004). Essential oils: their antibacterial properties and potential applications in foods-a review. Int. J. Food Microbiol. 94 (3), 223-253.

5. Elamrani A., Zsira S., Benjilali B., Berrada M. (2000). A study of Moroccan Rosemary oils. J.Essent. Oil Res., 12, 487-495.

6. Eloutassi N. (2004). Valorisation de Rosmarinus officinalis marocain par des procédés biotechnologiques. Thèse de doctorat, Laboratoire de Biotechnologie, Faculté des sciences, Université Sidi Mohammed Ben Abdellah, Fès, Maroc.

7. Eloutassi N., Louasté B., Boudine L., Remmal A. (2013). Contribution au développement des régions rurales : Conservation de Rosmarinus officinalis. ScienceLib. Envir. V 5, 130409, pp 1 -13.

8. Granger R., Passet J., Arbousset G. (1976). Activité optique de l'essence de romarin, Rosmarinus officinalis L. La France et ces parfums. $\mathrm{N}^{\circ} 67,62$.

9. Herrera J. (2005). Flower size variation in Rosmarinus officinalis: individuals, populations and habitats. Annals of Botany. 95:431-437.

10. L'Etablissement Autonome de Contrôle et de Coordination des Exportations 2014

11. M. Minaiyan, A.R. Ghannadi, M. Afsharipour, P. Mahzouni (2011). Effects of extract and essential oil of Rosmarinus officinalis L. on TNBS-induced colitis in rats Res Pharm Sci, 6, pp. 13-21

12. Mateu A.I., Aguilella A., Boisset F., Currás R., Guara M., Laguna E., Marzo A., Puche F., Pedrola J. (2013). Geographical patterns of genetic variation in rosemary (Rosmarinus officinalis) in the Mediterranean basin. Botanical Journal of the Linnean Society. V $171,4,700-712$.

13. Mehdioui R., Kahouadji A. (2007). Etude ethnobotanique auprès de la population riveraine de la forêt d'Amsittène : cas de la Commune 
d'Imi n'Tlit (Province d'Essaouira). Bulletin de l'Institut Scientifique, Rabat, section Sciences de la Vie. n²9, 11-20.

14. P.C. Dias, M.A. Foglio, A. Possenti, J.E. de Carvalho (2000). Antiulcerogenic activity of crude hydroalcoholic extract of Rosmarinus officinalis L J Ethnopharmacol, 69, pp. 57-62

15. Ph. Eur., (2014). Pharmacopée Européenne est la référence juridique et scientifique en matière de normes de pharmacopée en Europe. Elle contribue à assurer l'accès à des médicaments de qualité sur l'ensemble du continent, et au-delà. 8éme édition (2 220 monographies et 340 chapitres généraux et méthodes d'analyse, ainsi que 2500 descriptions de réactifs).

16. Wang, W., Wu, N., Zu, Y.G., Fu, Y.J., (2008). Antioxidative activity of Rosmarinus officinalis L. essential oil compared to its main components. Food Chem. 108 (3), 1019-1022. 